\title{
R-spondin 2 promotes proliferation and migration via the Wnt/ $\beta$-catenin pathway in human hepatocellular carcinoma
}

\author{
XINGUANG YIN ${ }^{1,2}$, HUIXING YI ${ }^{3}$, LINLIN WANG ${ }^{4}$, WANXIN WU ${ }^{5}$, XIAOJUN WU $^{1}$ and LINGHUA YU ${ }^{1}$ \\ ${ }^{1}$ Centre for Gastroenterology and Hepatology, The First Affiliated Hospital of Jiaxing College; \\ ${ }^{2}$ Centre for Gastroenterology and Hepatology, The Maternity and Child Health Care Hospital Affiliated to Jiaxing College, \\ Jiaxing, Zhejiang 314001; ${ }^{3}$ Intensive Care Unit, The Second Affiliated Hospital of Zhejiang University, \\ Hangzhou, Zhejiang 310009; ${ }^{4}$ Department of Basic Medicine Sciences, School of Medicine, Zhejiang University, \\ Hangzhou, Zhejiang 310058; ${ }^{5}$ Deparment of Pathology, The First Affiliated Hospital of Jiaxing College, \\ Jiaxing, Zhejiang 314001, P.R. China
}

Received March 6, 2016; Accepted February 13, 2017

DOI: $10.3892 / \mathrm{ol} .2017 .6339$

\begin{abstract}
Hepatocellular carcinoma (HCC) is a leading cause of malignant disease-associated mortality, particularly in China. The RSPO2 (R-spondin 2) gene is evolutionarily conserved in vertebrates and is involved in developmental and physiological processes. Importantly, RSPO2 has been reported to be associated with colon cancer and potentiate the Wnt/ $\beta$-catenin signaling pathway. In the present study, enhanced expression of RSPO2 in HCC was observed using tissue microarray. Similarly, the expression level of RSPO2 was higher in HepG2, Huh7 and Hep3B cells but lower in Bel7404 and QGY7703 cells compared with human normal QSG7701 liver cells. Subsequently, gain-of-function studies indicated that RSPO2 promotes the proliferation and migration of QGY7703 cells based on lentivirus-based gene delivery. Furthermore, it was revealed that p21 and leptin, rather than vascular endothelial growth factor-A, are involved in the function of RSPO2 in QGY7703 cells. Particularly, the signal transducer and activator of transcription 3 (STAT3) and $\mathrm{Wnt} / \beta$-catenin signaling pathways are involved in this process. Overexpression of RSPO2 resulted in the elevated expression of phosphorylated STAT3, $\beta$-catenin and c-Myc. Therefore, the present study is beneficial to the understanding of RSPO2-involved liver cancer transformation and drug discovery.
\end{abstract}

Correspondence to: Professor Linghua $\mathrm{Yu}$, Centre for Gastroenterology and Hepatology, The First Affiliated Hospital of Jiaxing College, 1882 Central-South Road, Jiaxing, Zhejiang 314001, P.R. China

E-mail: yu.1h70s@gmail.com

Key words: hepatocellular carcinoma, R-spondin 2, Wnt, $\beta$-catenin

\section{Introduction}

Hepatocellular carcinoma (HCC) is one of the most common cancer types and a major cause of cancer-associated mortality worldwide (1). The majority of HCC cases are caused by chronic hepatitis B or hepatitis C infections (2). HCC, characterized by rapid recurrence and poor survival, remains a challenging disease to treat (3). As HCC is not sensitive to radiotherapy or chemotherapy, surgery is the only effective treatment (4). However, the rate of recurrence is high and metastasis is common following surgery, which leads to the poor prognosis for HCC (3). Therefore, it is necessary to understand the molecular mechanisms underlying the growth and metastasis of HCC, which may help to identify effective diagnosis and therapeutic targets to improve the survival. However, the associated molecular mechanisms of HCC progression are not well understood.

The R-spondin (RSPO) protein family consists of four homologous members, which are evolutionarily conserved in vertebrates and are involved in a broad range of developmental and physiological processes $(5,6)$ : RSPO1 is important for sex determination (7); RSPO2 is required for limb, laryngeal-tracheal and lung development (8); RSPO3 is critical for placental formation (9); and the mutation of RSPO4 results in inherited anonychia (10). The association between RSPO and cancer has not been extensively studied. It has been reported that RSPO2 and RSPO3 insertional activation is observed in the mouse mammary tumor virus model system $(11,12)$. Administration of RSPO1 protein to mice induces rapid crypt cell proliferation, which causes a marked increase in the size of the small intestine (13). Seshagiri et al (14) identified that RSPO2 and RSPO3 transcript fusion occurs in $10 \%$ of colon tumors. In addition, it was found that RSPO fusions occur exclusively in tumors without adenomatous polyposis coli mutations, indicating that RSPO genes have a role in the activation of Wnt signaling and tumorigenesis (14). Subsequently, RSPO gene fusions were also observed in a subset of colon tumors in the Japanese population, and forced expression 
of the RSPO gene was revealed to increase the growth of colorectal cells (15).

A number of previous studies have indicated that RSPOs may potentiate Wnt signaling via stimulation of the leucine-rich repeat-containing G-protein coupled receptors (LGR) 4, LGR5 and LGR6 (16-18). Wnt proteins, comprising a large family of extracellular, lipid-modified glycoproteins, are crucial for embryonic development and cell proliferation, regulation, differentiation, survival and tissue homeostasis in adults $(19,20)$. It was demonstrated that RSPOs cooperate with Wnts during development, particularly by promoting the transcriptional activity of $\beta$-catenin, which is an important mechanism of the Wnt signaling pathway $(5,16)$. It has been revealed that canonical $\mathrm{Wnt} / \beta$-catenin signaling serves an important role in numerous cancer types, including lung, breast, brain, colorectal and liver tumors (21-23). Interaction between RSPOs and LGR4, as well as the intracellular signaling proteins, promotes phosphorylation of LRP5/6, stabilizes $\beta$-catenin expression and promotes its transcriptional activity (18). Activation of $\mathrm{Wnt} / \beta$-catenin results in the increased expression level of its target genes, including cyclin D1 and c-Myc, which are important for driving tumorigenesis in numerous types of cancer (24).

In the present study, high expression of RSPO2 was observed in certain HCC cell lines. Tissue microarray also revealed that the expression of RSPO2 is increased in primary tumors compared with the adjacent normal tissues. Functional study revealed that overexpression of RSPO2 enhances the cell proliferation and anchorage-independent growth of the human liver QGY7703 cancer cell line. In addition, RSPO2 overexpression may promote cell motility, which was demonstrated using Transwell and wound healing assays. Similar to a previous study (25), the present study also revealed that overexpression of RSPO 2 is involved in Wnt/ $\beta$-catenin activation via increasing the expression of $\beta$-catenin and the downstream gene c-Myc. The present study revealed the functional role of RSPO2 in HCC and indicated that RSPO2 may be a potential drug target for patients with liver tumors.

\section{Materials and methods}

Patients and liver tissue samples. A total of 72 human liver tissues were obtained from 24 patients with HCC who had undergone surgical resection between January 2013 and December 2014 at the First Affiliated Hospital of Jiaxing College (Jiaxing, China) with written informed consent and ethical approval from the Local Ethics Committee of Jiaxing College. Patients did not receive radiotherapy or chemotherapy prior to surgery.

Tissue microarray. Tissue samples (24 tumor tissues, 24 paired non-tumor adjacent tissues and 24 normal tissues from 24 HCC patients) were formalin-fixed and paraffin-embedded. The samples were used to construct tissue microarrays (TMAs) using a Beecher Instrument (Sun Prairie, WI, USA) as described previously (26). A total of 3 tissue cylinders of $0.6 \mathrm{~mm}$ in diameter were punched from each sample. Subsequent to sectioning ( $4 \mathrm{~mm}$ for each section), tissue slides were baked at $60^{\circ} \mathrm{C}$ for $2 \mathrm{~h}$, and kept at $4^{\circ} \mathrm{C}$ for subsequent analysis. TMAs were stained with hematoxylin and eosin ( $\mathrm{H} \& \mathrm{E})$. A trained pathologist reevaluated $\mathrm{H} \& \mathrm{E}$-stained samples to determine the tumor stage and grade according to the WHO criteria (27). The slides with tissue sections were subjected to immunohistochemical detection of RSPO2, using the primary antibody against RSPO2 (dilution, 1:50; cat. no. ab73761; Abcam, Cambridge, MA, USA). Following rinsing with $1 \mathrm{X}$ PBS, slides were incubated with biotinylated anti-rabbit $\mathrm{IgG}$ for $30 \mathrm{~min}$ (dilution, 1:1,000) using Vectastain Elite ABC kit (cat. no. PK-6100; Vector Laboratories, Inc., Burlingame, CA, USA). The detection was achieved using the avidin-biotin peroxidase method with a diaminobenzidine chromogen kit (cat. no. SK-4100; Vector Laboratories, Inc.). The TMAs were evaluated for RSPO2 expression by a trained pathologist and were scored as strong $(+++)$ with $>80 \%$ positive cells, moderate $(++)$ with $30-80 \%$ positive cells, weak $(+)$ with observed $<30 \%$ stained cells or absent (-). The subcellular localization of the staining was noted for each score using CI microscopy by NIS-Elements F under x100 magnification.

Cell culture. The QSG-7701 cell line derived from human normal liver tissue and the human hepatoma cell lines Bel7404, QGY7703, HepG2, Huh7, 293T and Hep3B were purchased from the Cell Bank of the Type Culture Collection of the Chinese Academy of Sciences (Shanghai, China). QSG-7701 and QGY7703 cells were cultured in RPMI-1640 (cat. no. SH30809.01; HyClone; GE Healthcare Life Sciences, Logan, UT, USA) medium containing $10 \%$ fetal bovine serum (FBS) (cat. no. 10099141; Gibco; Thermo Fisher Scientific, Inc., Waltham, MA, USA). Bel7404, HepG2, Huh7 and Hep3B cells were cultured in Dulbecco's modified Eagle's medium (DMEM; cat. no. SH30243.01B; HyClone; GE Healthcare Life Sciences) with high glucose, supplemented with 10\% FBS. All cells were cultured in an atmosphere of $95 \%$ air and $5 \% \mathrm{CO}_{2}$ under humidified conditions.

Western blot analysis. All cultured cells were lysed using radioimmunoprecipitation assay lysis and extraction buffer (cat. no. 89900; Thermo Fisher Scientific, Inc.). Total proteins were subjected to concentration determination using a commercial bicinchoninic acid quantification kit, according to the manufacturer's protocol (cat. no. 23227; Thermo Fisher Scientific, Inc.).

Lysates from QGY7703 cells or HCC tissue $(15 \mu \mathrm{g})$ were subjected to $12 \%$ SDS-PAGE for protein separation and then electrophoretically transferred to nitrocellulose membranes (Axygen Scientific, Union City, CA, USA). Subsequent to being blocked by PBS containing 5\% fat-free milk, the nitrocellulose membranes were incubated with rabbit polyclonal antibody for RSPO2 (cat. no. ab73761; dilution, 1:1,000; Abcam), $\beta$-catenin (cat. no. 8480; dilution, 1:1,000; Cell Signaling Technology, Inc., Danvers, MA, USA), c-Myc (cat. no. 5605; dilution, 1:1,000; Cell Signaling Technology, Inc.), vascular endothelial growth factor (VEGF)-A (cat. no. ab51745; dilution, 1:1,000; Abcam), phosphorylated signal transducer and activator of transcription 3 (p-STAT3) (cat. no. 9130; dilution, 1:1,000; Cell Signaling Technology, Inc.), leptin (cat. no. ab3583; dilution, 1:1,000; Abcam), p21 (cat. no. SC-397; dilution, 1:500; Santa Cruz Biotechnology, Inc., Dallas, TX, USA) and rabbit polyclonal antibody for $\beta$-actin (cat. no. 10303001; 
dilution, 1:5,000; Harmonious One Biotechnology, Shanghai, China) overnight at $4^{\circ} \mathrm{C}$ and then incubated with horseradish peroxidase-conjugated rabbit IgG (cat. no. 7074; dilution, 1:3,000; Cell Signaling Technology, Inc.) for $1.5 \mathrm{~h}$ at room temperature. The immunolabeled proteins were detected using a commercial enhanced chemiluminescent detection kit (cat. no. 108070002; Harmonious One Biotechnology, Shanghai, China). Results were quantified by a luminescent digital image analyzer Bio-Spectrum600 (UVP; Upland, CA, USA). Band intensity was assessed using a Gel-Pro analyzer (V6.3; Media Cybernetics, Inc., Rockville, MD, USA).

Lentivirus vector construction. The total RNA from $5 \times 10^{6}$ HepG 2 cells was extracted using the phenol-chloroform method following TRIzol (cat. no. 15596-026; Invitrogen; Thermo Fisher Scientific, Inc.) lysis, according to the manufacturer's protocol. The cDNA was prepared by reverse transcription using a random primer (D3801; Takara Biotechnology Co., Ltd., Dalian, China) at $37^{\circ} \mathrm{C}$ for $1 \mathrm{~h}$, following the denaturation of total RNA by heating for $5 \mathrm{~min}$ at $37^{\circ} \mathrm{C}$, followed by immediate chilling on ice. The full coding region of the RSPO2 gene was isolated following denaturation at $95^{\circ} \mathrm{C}$ for $5 \mathrm{mint}, 36$ cycles of $95^{\circ} \mathrm{C}$ for $30 \mathrm{sec}, 56^{\circ} \mathrm{C}$ for $30 \mathrm{sec}$ and $72^{\circ} \mathrm{C}$ for $45 \mathrm{sec}$, and then subjected to post-elongation for $10 \mathrm{~min}$ at $72^{\circ} \mathrm{C}$. The mixture for polymerase chain reaction (PCR) was composed of $5 \mu \mathrm{l}$ of 10X PCR buffer, $0.2 \mathrm{mM}$ dNTP, $0.2 \mu \mathrm{M}$ RSPO2-EcoRI-F primer (5'-CCGGAATTCATGCAG TTTCGCCTTTTCTC-3'), $0.2 \mu \mathrm{M}$ RSPO2-BamHI-R primer (5'-CGCGGATCCTTATTGGTTAGCTCTGTCTGTAGC-3'), 2 U PFU polymerase (cat. no. 101060002; Harmonious One Biotechnology) and sterile distilled water. The human RSPO2 gene was then subcloned into EcoRI and BamHI sites of the lentiviral vector pLV-mCherry (2A) puro (cat. no. VL3405; Inovogen Biotechnology Pvt. Ltd., Beijing, China) with the selective marker gene puromycin by a classic ligation and transformation method using T4 ligase (cat. no. EL0011; Fermentas; Thermo Fisher Scientific, Inc.) and DH5 $\alpha$ chemical competent E. coli cells (cat. no. C502-03; Vazyme, Piscataway, NJ, USA).

Lentivirus production and cells transduction. Packaging of pseudotyped recombinant lentivirus was performed by transfection of $293 \mathrm{~T}$ cells. Briefly, $1.5 \times 10^{6} 293 \mathrm{~T}$ cells were plated in a $6-\mathrm{cm}$ dish and cultured for $20 \mathrm{~h}$. The cells were then cotransfected with either $1.7 \mu \mathrm{g}$ pLV-mCherry (2A) puro or pLV-mCherry (2A) puro-RSPO2, $1.13 \mu \mathrm{g}$ pCMV $\Delta 8.91$ and $0.57 \mu \mathrm{g}$ pMD.G (a gift from Institute of Biochemistry and Cellular Biology, Shanghai, China) using Lipofectamine 2000 (Invitrogen; Thermo Fisher Scientific, Inc.) following the manufacturer's protocol. The supernatant containing the lentivirus was harvested at $72 \mathrm{~h}$ and filtered through a $0.45 \mu \mathrm{m}$ low protein binding polysulfonic filter (EMD Millipore, Billerica, MA, USA). QGY7703 cells were inoculated in 6-well plates in advance at a density of $2 \times 10^{5}$ cells per well and presented with $\sim 40 \%$ confluence following incubation for $20 \mathrm{~h}$ at $37^{\circ} \mathrm{C}$. The cells were then infected with $1 \mathrm{ml}$ lentivirus suspension in the presence of $8 \mu \mathrm{g} / \mathrm{ml}$ polybrene (Chemicon; EMD Millipore). Following transduction for $48 \mathrm{~h}$ at $37^{\circ} \mathrm{C}$, QGY7703 cells were selected with $2.0 \mu \mathrm{g} / \mathrm{ml}$ puromycin for 10 days when all the blank control cells without transfection were eradicated. The selected cells were used for growth, anchorage-independent growth and migration assays.

Viability analysis. The human hepatoma QGY7703 cell line with stable overexpression of RSPO2 and the control QGY7703 cells transfected with empty vectors were seeded onto 96-well plates at the density of $2.0 \times 10^{3}$ per well. Cells were analyzed using an MTT assay at day 1, 2, 3, 4 and 5 subsequent to cell seeding. Briefly, $100 \mu \mathrm{g}$ of MTT (cat. no. 0793-1G; Amresco, LLC, Solon, OH, USA) was added to each well. Following incubation for $4 \mathrm{~h}$ at $37^{\circ} \mathrm{C}$, the purple formazan crystals generated from viable cells were dissolved by adding $100 \mu \mathrm{l}$ of dimethyl sulfoxide to each well. The absorbance of each well was then read at $570 \mathrm{~nm}$.

Anchorage-independent growth analysis. QGY7703 cells transfected with pLV-mCherry (2A) puro-RSPO2 or pLV-mCherry (2A) puro were trypsinized and suspended in culture medium. The cells were seeded on each well of a 24-well plate with complete medium at the density of $1.0 \times 10^{3}$ cells. Following growth for 10 days, the cells were subjected to fixation by methanol for $10 \mathrm{~min}$ and then stained by $0.1 \%$ crystal violet at room temperature for $30 \mathrm{~min}$. Following removal of the dye, colonies containing $>50$ cells were counted in five random fields using TiS microscopy by NIS-Elements Viewer (version 4.2; Nikon, Tokyo, Japan) under x100 magnification.

Migration assay. A 24-well Transwell chamber with $8.0 \mu \mathrm{m}$ pore size (Costar; Corning Incorporated, Corning, NY, USA) was used for the migration assay. The pre-balance of the Transwell chamber was performed by adding DMEM without FBS into the upper and bottom chamber overnight at $37^{\circ} \mathrm{C}$. QGY7703 cells transfected with pLV-mCherry (2A) puro-RSPO2 or pLV-mCherry (2A) puro were trypsinized and suspended in $200 \mu \mathrm{l}$ serum-free medium and were seeded in the upper chamber at a density of $1.5 \times 10^{5}$ cells per well. The bottom chamber was filled with DMEM containing $10 \%$ FBS. Following incubation for 24 and $48 \mathrm{~h}$ at $37^{\circ} \mathrm{C}$, the non-migrating cells were removed by soft scratch with small cotton swabs and rinsed with 1 XPBS. Migrated cells were then dried, fixed with methanol and stained with $0.1 \%$ crystal violet at room temperature for $30 \mathrm{~min}$. The transmembrane cells were counted using a TiS microscopy by NIS-Elements Viewer (version 4.2, Nikon) under x100 magnification.

Wound healing assay. RSPO2-overexpressed QGY7703 cells or control cells were plated onto a 24 -well plate with $4 \times 10^{6}$ per well and incubated for $24 \mathrm{~h}$ at $37^{\circ} \mathrm{C}$. Linear scratch wounds were then produced using a $10 \mu \mathrm{l}$ pipette tip on the confluent cell monolayer. The medium was replaced with the serum-free medium. Images were captured at $0,12,24,36$ and $72 \mathrm{~h}$ and the wounding size was quantified and analyzed by NIS-Elements Viewer (version 4.2, Nikon).

Statistical analysis. Data are presented as the mean \pm standard deviation. A two-tailed Student's t-test was employed to evaluate the differences between groups. $\mathrm{P}<0.05$ was considered to indicate a statistically significant. The differences of indexes between tumor tissues and paired non-tumor adjacent tissues 


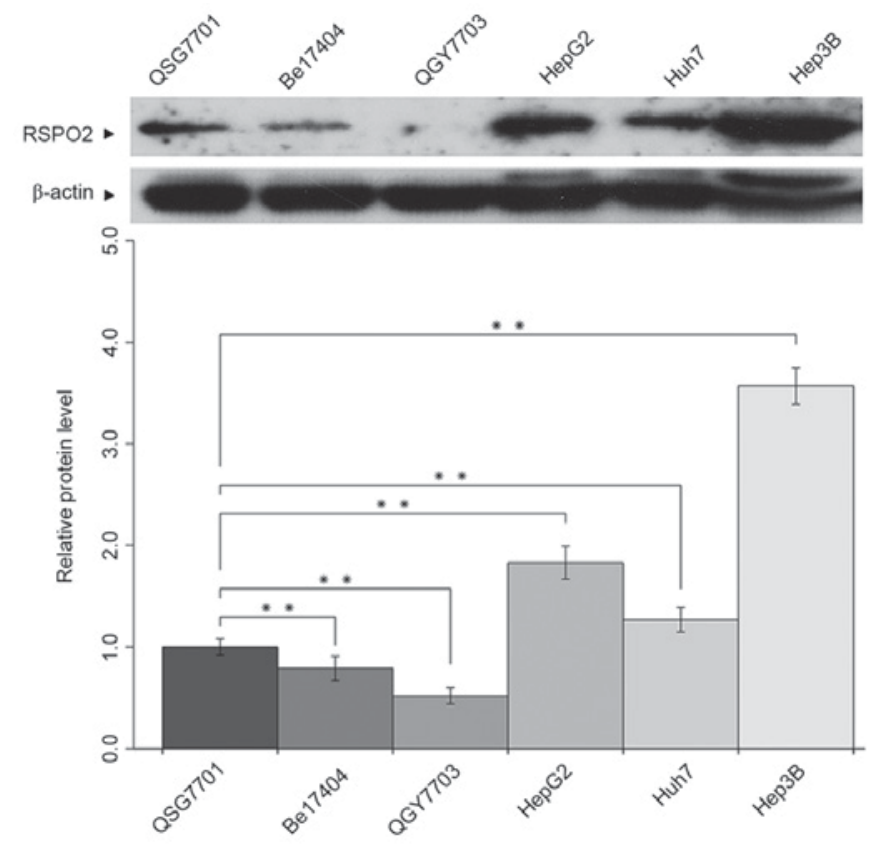

Figure 1. RSPO2 expression in various hepatocellular carcinoma cells Immunoblotting of RSPO2 in various hepatocellular carcinoma cell lines $\beta$-actin was used as a loading control. Data are presented as the mean of three independent experiments, and error bars are standard deviation of means. ${ }^{* *} \mathrm{P}<0.01$, compared with the control. RSPO2, R-spondin 2 .

were analyzed using the Wilcoxon signed-rank test. Differences between groups were analyzed by the Mann-Whitney U test. Data were processed with R Studio (v1.0; Boston, MA, USA).

\section{Results}

Expression of RSPO2 in various HCC cells. The expression level of RSPO2 was detected in various HCC cells by western blot analysis. As presented in Fig. 1, increased expression levels of RSPO2 were observed in HepG2, Huh7 and Hep3B cells compared with the human normal liver QSG-7701 cell line. However, markedly decreased expression of RSPO2 was found in the human hepatoma Bel7404 and QGY7703 cell lines compared with QSG-7701 cells.

Overexpression of RSPO2 in liver cancer tissues. The expression of RSPO2 in clinical samples of HCC was analyzed. A total of 24 pairs of human hepatic carcinoma and matched adjacent non-tumor tissues or normal tissues were examined using immunohistochemical staining with an antibody against human RSPO2. Samples were considered RSPO2-positive if either the cell nucleus or cytoplasm stained positive. As presented in Fig. 2A and B, the staining of RSPO2 was primarily observed in the cytoplasm of cancer cells. Fig. 2A presents representative examples from the tissue microarray for each RSPO2 staining score, ranging from 0 to +++ . Intense expression of RSPO 2 in tumor tissue (score ++ or +++ ) was identified in 16/24 patients $(66.7 \%)$, whereas in other patients $(33.3 \%)$ a weak immunoreactivity (score + ) was detected. However, the expression of RSPO2 in non-tumor adjacent tissues was significantly lower compared with tumor tissues (Wilcoxon signed-rank test, $\mathrm{P}=0.007$ ). Moderate expression of RSPO2 (score ++ ) in
Table I. Characteristics and RSO2 expression of patients with hepatocellular carcinoma.

\begin{tabular}{|c|c|c|c|c|}
\hline \multirow[b]{2}{*}{ Characteristics } & \multirow[b]{2}{*}{$\begin{array}{c}\text { Patients, } \\
\mathrm{n}(\%)\end{array}$} & \multicolumn{2}{|c|}{ RSPO2 expression } & \multirow[b]{2}{*}{ P-value } \\
\hline & & $\begin{array}{l}\text { Absent or } \\
\text { weak, } n\end{array}$ & $\begin{array}{l}\text { Moderate } \\
\text { to high, } \mathrm{n}\end{array}$ & \\
\hline Age & & & & 0.155 \\
\hline$\leq 50$ years & $13(54)$ & 6 & 7 & \\
\hline$>50$ years & $11(46)$ & 2 & 9 & \\
\hline Gender & & & & 0.766 \\
\hline Male & $18(75)$ & 7 & 11 & \\
\hline Female & $6(25)$ & 1 & 5 & \\
\hline Tumor grading & & & & 0.286 \\
\hline I & $3(3)$ & 0 & 3 & \\
\hline II & $19(79)$ & 7 & 12 & \\
\hline III & $2(8)$ & 1 & 1 & \\
\hline Tumor staging & & & & 0.996 \\
\hline II & $4(17)$ & 1 & 3 & \\
\hline III & $20(83)$ & 7 & 13 & \\
\hline
\end{tabular}

P-value calculated using Mann-Whitney U test. RSPO2, R-spondin 2.

non-tumor adjacent tissue was found in 6 of 24 patients (25\%), whereas the remaining $75 \%$ exhibited weak expression of RSPO2 (score +). Similar expression patterns were found in the paired normal tissues. The expression of RSPO2 in the paired normal tissues was significantly lower compared with tumor tissues (Wilcoxon signed-rank test, $\mathrm{P}=0.001$ ). Representative images of RSPO2 expression in the paired normal, adjacent and tumor tissues are shown in Fig. 2A.

Statistical analyses were performed to examine the association between RSPO2 expression and the clinicopathological characteristics of hepatic carcinoma. As shown in Table I, no association was observed between the expression of RSPO2 and patient age or gender, tumor grading and tumor staging in patients with HCC. A small sample size $(n=24)$ may be the reason that no statistically significant results were identified.

RSPO2 overexpression enhances the proliferation and anchorage-independent growth of QGY7703 cells. To define the function of RSPO2 in HCC, RSPO2 overexpression in the QGY7703 cell line was achieved by lentivirus delivery (Fig. 3A). The cell growth of QGY7703 cells with stable overexpression of RSPO2 was firstly evaluated by MTT assay. RSPO2 overexpression significantly promoted QGY7703 cell growth at days 2, 3, 4 and 5 compared with the control group, in which QGY7703 cells were transfected with empty vector $(\mathrm{P}<0.01$; Fig. 3B).

Anchorage-independent growth was also used to examine whether the RSPO2 gene affects the tumorigenic growth of QGY7703 cells. As shown in Fig. 3C, RSPO2 overexpression significantly enhances soft agar growth of QGY7703 cells, as evidenced by the decrease in colony number and size compared with the control QGY7703 cells that were infected with the pLV-mCherry $(2 \mathrm{~A})$ puro empty vector $(\mathrm{P}<0.01)$. 
A

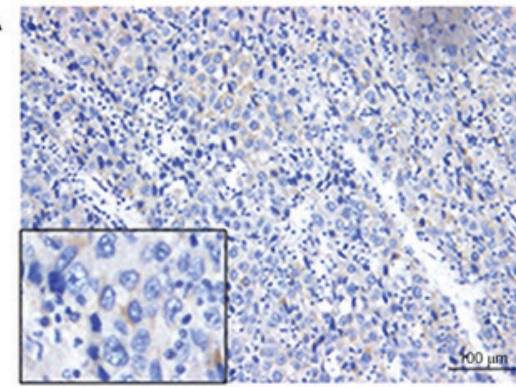

a

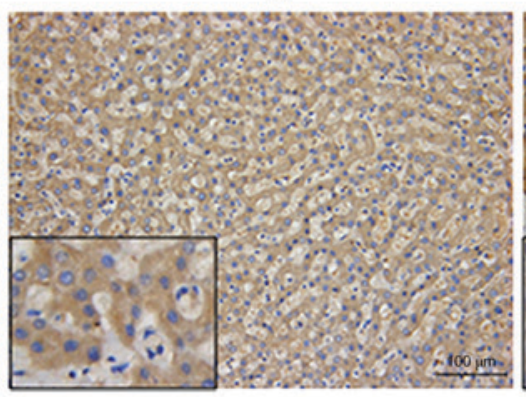

C

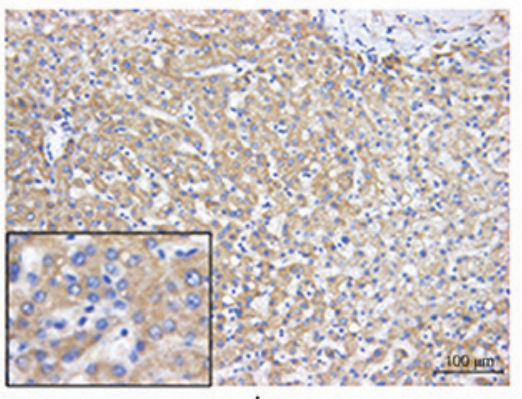

b

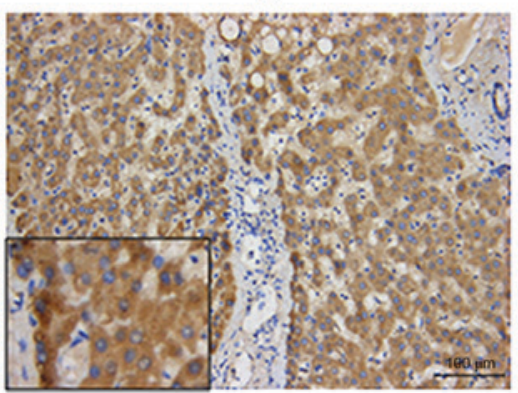

d

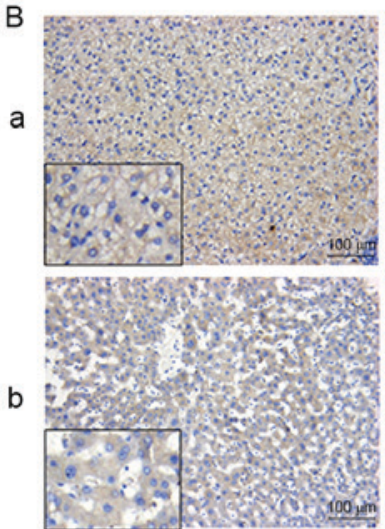

Normal
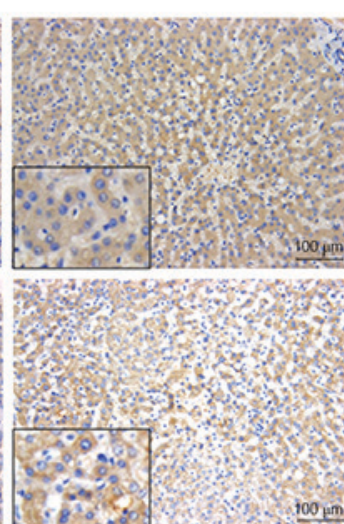

Adjacent
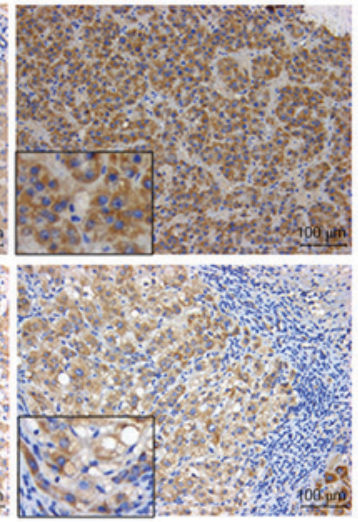

Tumor
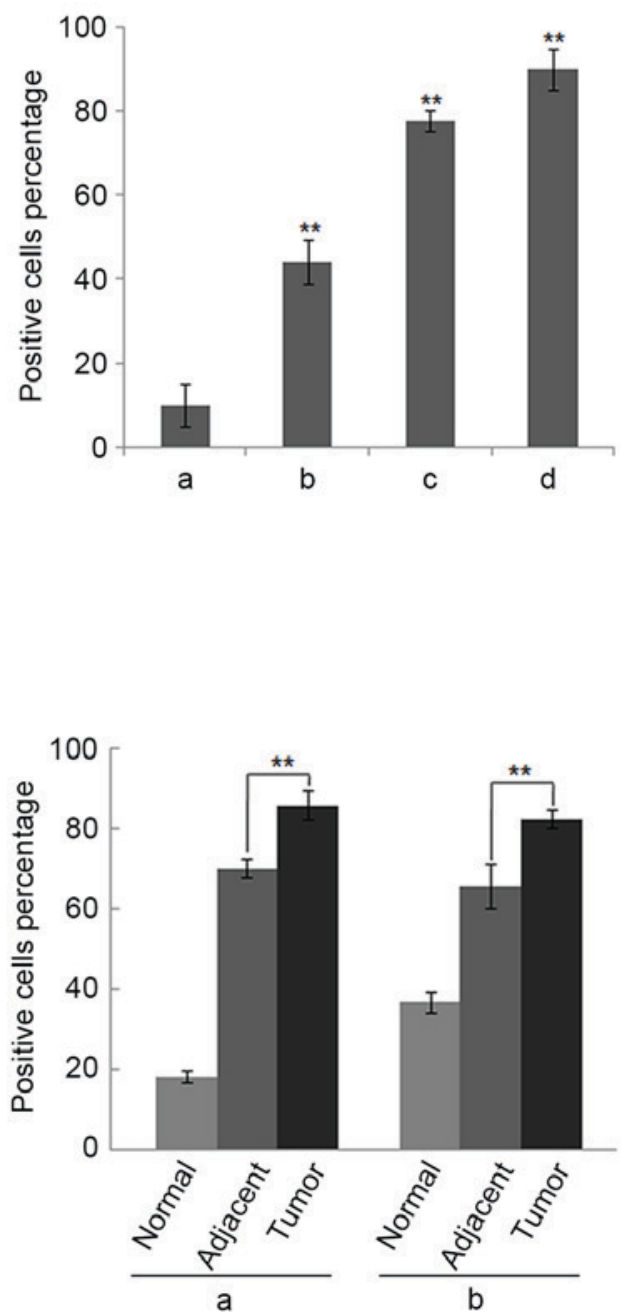

Figure 2. Immunohistochemical staining of RSPO2 in hepatocellular carcinoma tissue sections. (A) Immunohistochemical staining pattern of RSPO2. RSPO2 has a distinct immunoreactivity with pronounced intensity in the cytoplasm of cancer cells. Representative examples of (a) absent, (b) weak, (c) moderate and (d) intense expression are depicted. A representative part was shown with a magnification of 5 folds. (B) Representative images of RSPO2 expression in the normal, adjacent and tumor tissues from two patients (a) and (b). Weak expression of RSPO2 was observed in the normal and adjacent tissues, but the tumor tissues exhibited intense positive staining of RSPO2. A representative part was shown with a magnification of 5 folds. Data are presented as the mean of three independent experiments, and error bars are standard deviation of means. Scale bars, $100 \mu \mathrm{m} .{ }^{* *} \mathrm{P}<0.01$ compared with the control. RSPO2, R-spondin 2 .

RSPO2 overexpression promotes the migration of QGY7703 cells. To study the effect of RSPO2 on the motility of QGY7703 cells, Transwell and wound healing assays were performed. In the Transwell assay, the percentage of cells that migrated through the membrane was significantly increased in cells with RSPO2 overexpression compared with the control cells transfected with empty vector (Fig. 4A). The wound healing assay results also demonstrated that RSPO2 overexpression significantly enhanced the migration of QGY7703 cells compared with the control cells (Fig. 4B).

RSPO2 overexpression potentiates the activation of $W n t / \beta$-catenin. Previous studies have reported that the RSPO2 gene is involved in the activation of the Wnt $/ \beta$-catenin pathway. To improve the understanding of the biological role of the RSPO2 gene in HCC and the underlying mechanism of the aformentioned findings, the expression level of nuclear $\beta$-catenin was analyzed in QGY7703 cells. As shown in Fig. 5, the expression level of nuclear $\beta$-catenin was significantly increased in QGY7703 cells with stable overexpression of RSPO2 gene compared with the negative control group. The expression of c-Myc, one of the target genes of $\mathrm{Wnt} / \beta$-catenin signaling, was also analyzed. Consistently, c-Myc gene expression was significantly increased in QGY7703 cells with RSPO2 stable overexpression (Fig. 5). The present data indicated that overexpression of RSPO2 is involved in Wnt/ $\beta$-catenin activation via increasing the expression of $\beta$-catenin and its downstream genes.

RSPO2 regulates proliferation-associated genes and signaling pathways. To further elucidate the molecular mechanism underlying RSPO2-induced cell proliferation, the 
A
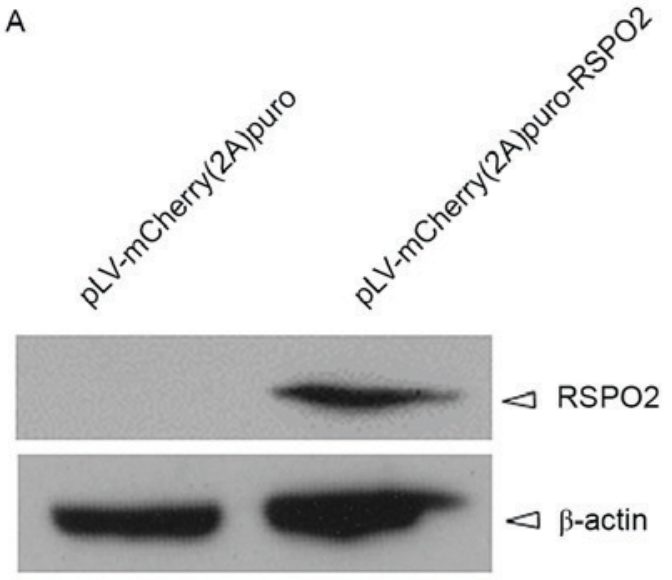

C

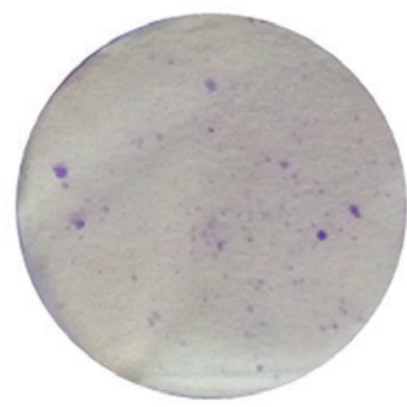

pLV-mCherry(2A)puro

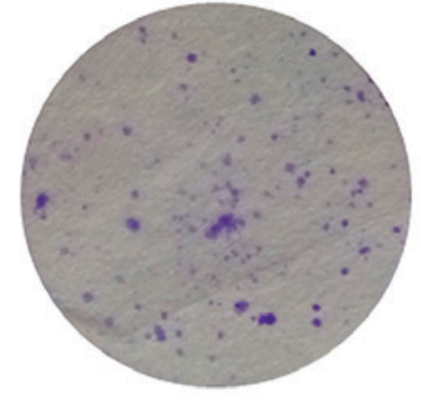

pLV-mCherry(2A)puro-RSPO2
B
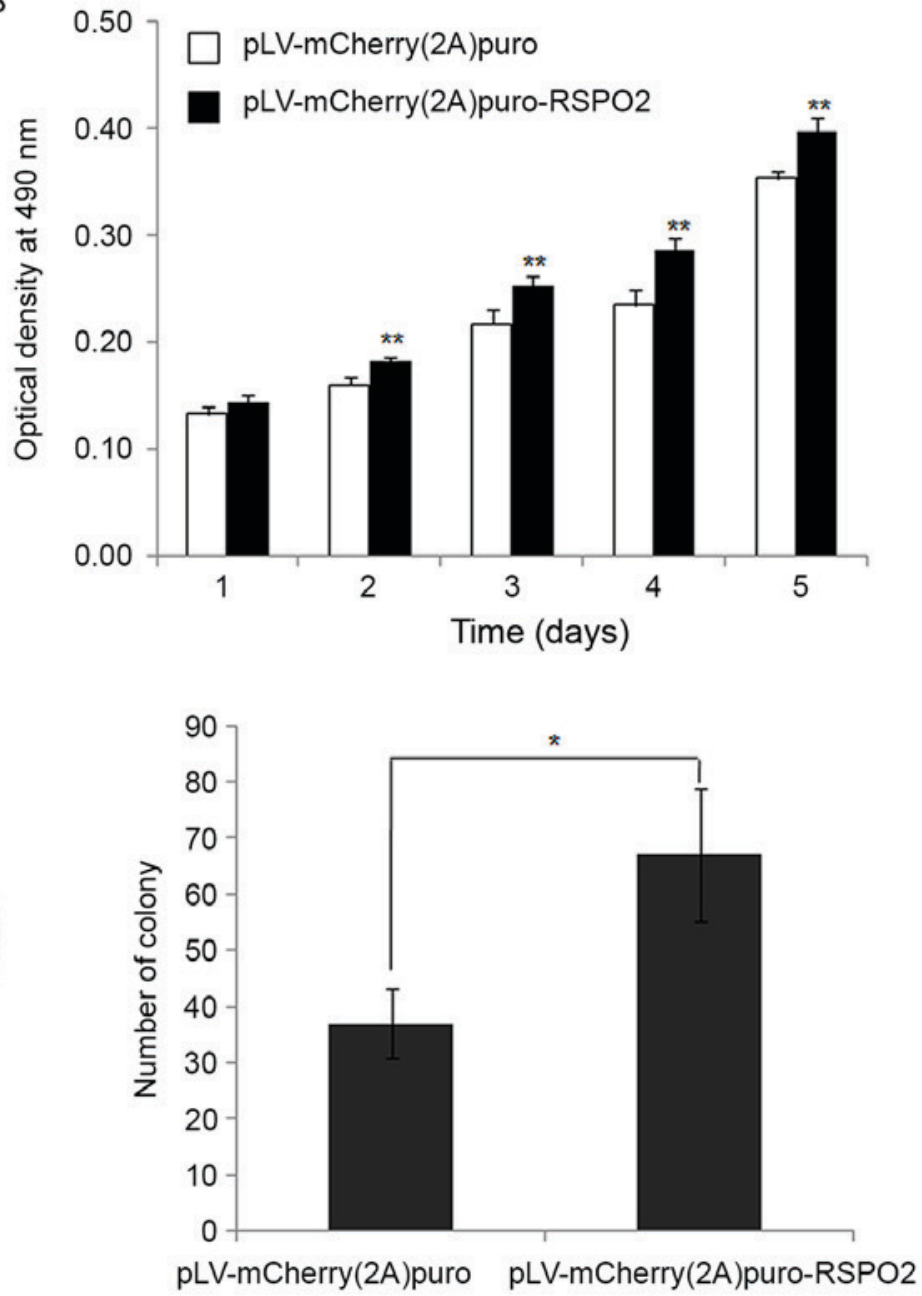

Figure 3. RSPO2 overexpression promotes the proliferation and anchorage-independent growth of the QGY7703 cell line. (A) Overexpression of RSPO2 in the QGY7703 cell line using immunoblotting analysis. $\beta$-actin was used as a loading control. (B) Cell growth of the QGY7703 cell line in response to RSPO2 gene overexpression. The MTT assay was employed to detect cell growth at 24, 48, 72, 96 and $120 \mathrm{~h}$ following cell seeding. (C) Effect of RSPO2 on colony formation of QGY7703 cells. Representative images are presented and the number of clones for each well were counted. Data are presented as the mean of three independent experiments, and error bars are standard deviation of means. ${ }^{*} \mathrm{P}<0.05 ;{ }^{* * *} \mathrm{P}<0.01$, compared with the control [pLV-mCherry(2A)puro]. RSPO, R-spondin 2.

proliferation-associated genes p21, leptin and VEGF-A, and the STAT3 signaling pathway were investigated. The p21 and leptin genes exhibited significantly reduced and increased expression, respectively (Fig. 5). However, the expression level of VEGF-A did not show a notable difference between control and RSPO2-overexpressed QGY7703 cells. The elevated expression of phosphorylated STAT3 indicated that the STAT3 signaling pathway may be involved in RSPO2-induced cell proliferation.

\section{Discussion}

The carcinogenesis of HCC is a multi-factorial, multi-step and complex process. Previous studies have documented that the bidirectional interactions between tumors and hepatic stellate cells (HSCs) compose an amplification loop to enhance metastatic growth in the liver $(28,29)$. Previous studies revealed that the RSPO family may promote HSC activation by enhancing the canonical Wnt pathway $(30,31)$. In the present study, the data provided evidence that RSPO2 expression may contribute to malignant biological behavior in HCC. Additional large-scale investigations are required to pinpoint the link between the expression level of RSPO2 and the clinical characteristics of human HCC. The present study demonstrated that the expression of RSPO2 is upregulated in various HCC cell lines. Paired HCC lesions and adjacent non-cancer tissues were found to express RSPO2 differently. The tumor tissues exhibited significantly increased expression of RSPO2 compared with adjacent non-tumor tissues. Furthermore, the present data demonstrated that RSPO2 overexpression enhances the cell proliferation and anchorage-independent growth of human liver QGY7703 cell lines. In addition, RSPO2 overexpression may also promote the motility of QGY7703 cells. Study of the underlying molecular mechanism indicated that overexpression of RSPO2 may be associated with Wnt/ $\beta$-catenin activation via increasing the expression of $\beta$-catenin and its 
A

pLV-mCherry(2A)puro

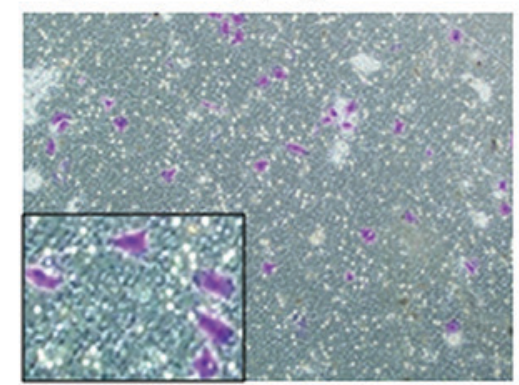

pLV-mCherry(2A)puro-RSPO2

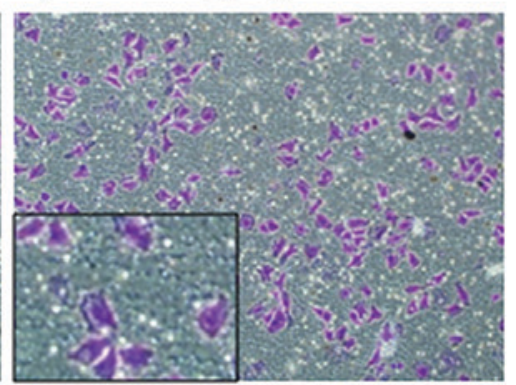

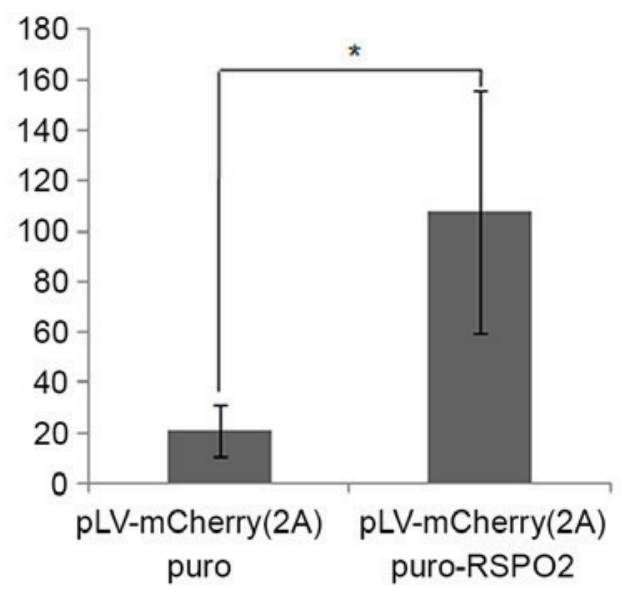

B
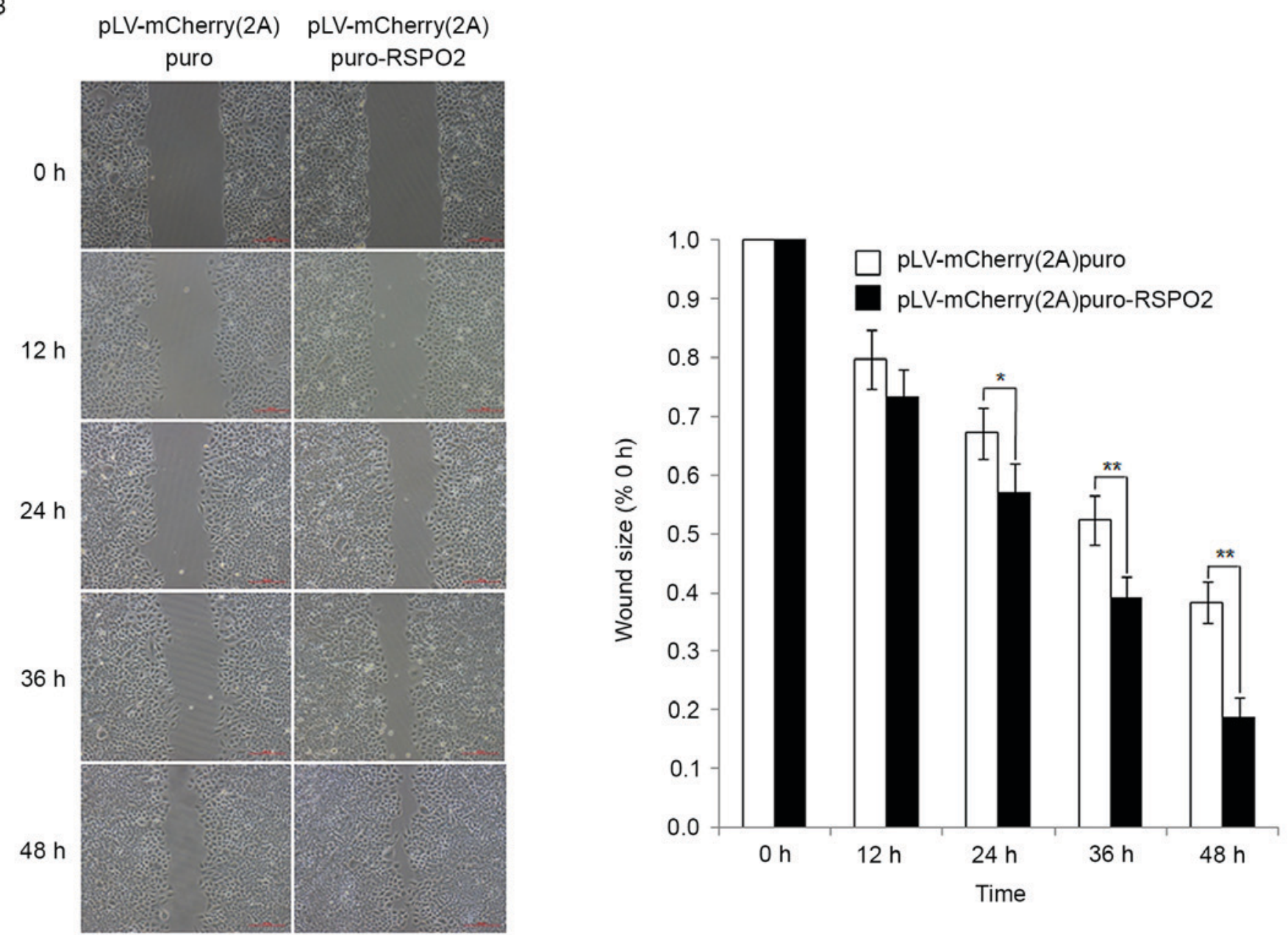

Figure 4. RSPO2 overexpression enhances the motility of the QGY7703 cell line. (A) Transwell migration assay of QGY7703 cells with stable overexpression of RSPO2 or empty vector. Representative images are presented and the number of migrated cells is shown. A representative part was shown with a magnification of 5 folds. Results are presented as the mean \pm SD of three independent experiments. (B) Wounding healing assay of QGY7703 cells with stable overexpression of RSPO 2 or empty vector. Images were taken at $0,12,24,36$ and $48 \mathrm{~h}$ following the scratch treatment. Representative images and quantitative analysis of relative wound size are shown. Data are presented as the mean of three independent experiments, and error bars are the SD of the means. "P $<0.05$; ** $\mathrm{P}<0.01$, compared with the control (pLV-mCherry(2A)puro). SD, standard deviation; RSPO2, R-spondin 2.

downstream gene c-Myc. Taken together, the present results revealed the potential functional role of RSPO2 in HCC and indicated that RSPO2 may be a potential drug target for patients with liver tumors.

Previous studies have revealed that RSPOs are involved in the activation of Wnt signaling, which is important for tumorigenesis $(14,32)$. However, the association between RSPO and cancer has not been extensively studied. Studies on the role of RSPO2 in cancer primarily focus on colon tumors $(14,15)$. A recent study reported that high-copy amplifications of RSPO2 gene were observed in $231 \mathrm{HCC}$ cases via whole exome sequencing (25). In the present 


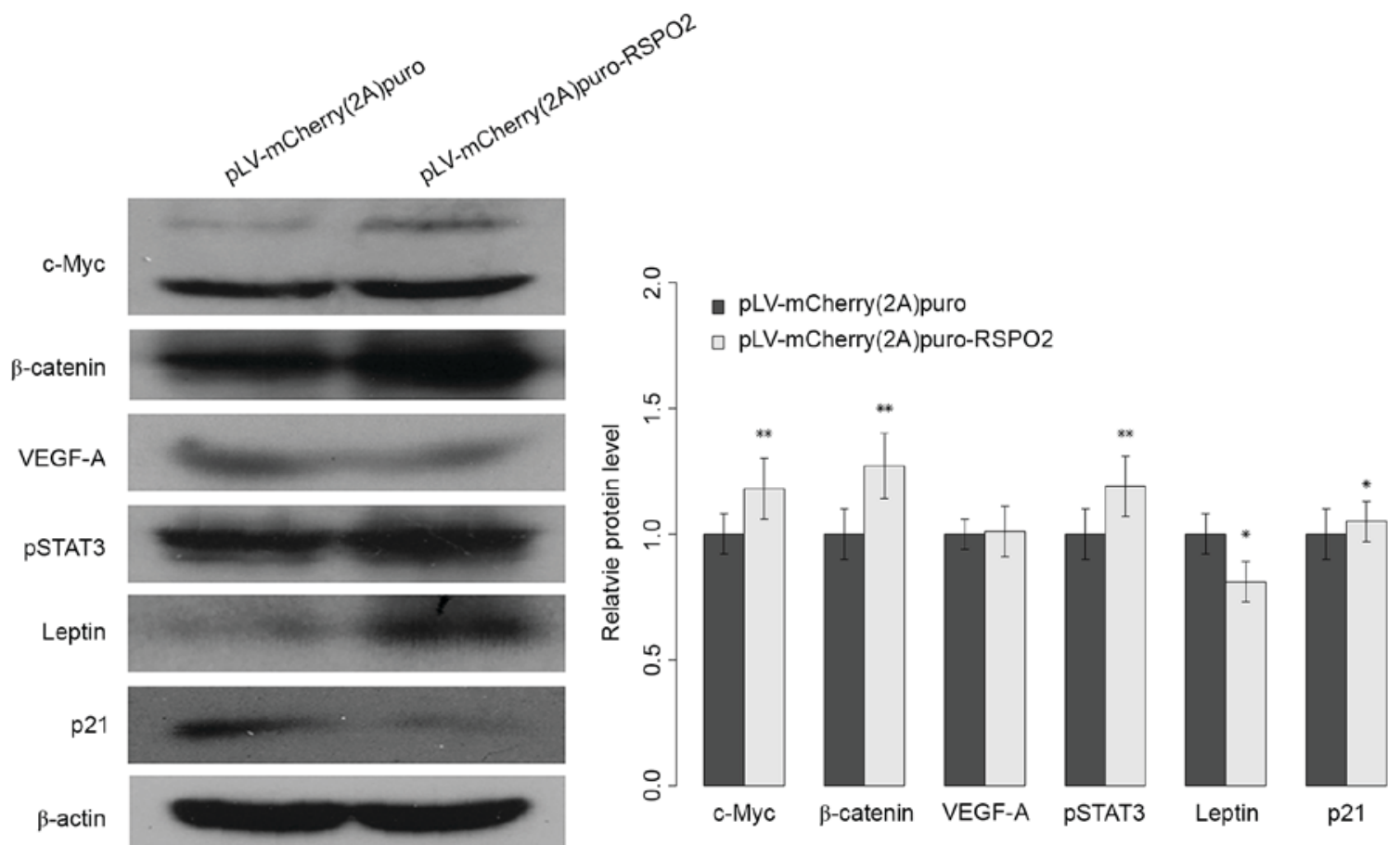

Figure 5. RSPO2 overexpression potentiates the activation of Wnt/ $\beta$-catenin. Western blot analysis of $\beta$-catenin, c-Myc, VEGF-A, pSTAT3, leptin and p21 in QGY7703 cells with stable overexpression of RSPO2 or empty vector. $\beta$-actin was used as the loading control. Data are presented as the mean of three independent experiments, and error bars are standard deviation of the means. "P<0.05; "* $\mathrm{P}<0.01$, compared with the control (pLV-mCherry (2A)puro). VEGF-A, vascular endothelial growth factor-A; pSTAT3, phosphorylated signal transducer and activator of transcription 3; RSPO2, R-spondin 2.

study, significantly increased expression levels of RSPO2 were detected in HCC tissues compared with the adjacent non-tumor tissues or paired normal tissue. Similarly, an increased expression level of RSPO2 was observed in HepG2, Huh7 and Hep3B cells compared with human normal liver QSG-7701 cell lines. High-copy amplifications of RSPO2 may aid the explanation of the phenomenon of increased expression of RSPO2 in tumor tissues.

In the present study, altered p21 and leptin expression levels were observed, which may contribute to RSPO2-induced proliferation on QGY7703 cells. Further work requires an elucidation of the spatial-temporal association between RSPO2 and p21 or leptin. Furthermore, systematic investigation of pivotal molecules located in the STAT3 signaling pathway may aid understanding of the role of RSPO2 in liver cancer transformation. Other signaling pathways may be examined to systematically elucidate this molecular mechanism, potentially contributing to identification of novel HCC therapeutics.

In conclusion, the present study revealed that $\mathrm{R}$-spondin 2 promotes proliferation and migration in various $\mathrm{HCC}$ cell lines via the $\mathrm{Wnt} / \beta$-catenin pathway. However, additional studies are required to confirm the tumor-promoting effects of $\mathrm{R}$-spondin 2 in mouse models.

\section{Acknowledgements}

The present study was funded by grants from the Jiaxing Municipal Science and Technology Project (grant no. 2014AY21030-1), the Zhejiang Science and Technology Public Welfare Project (grant no. 2015C33279), the Zhejiang Provincial Natural Science Fund (grant nos. LY16H030016 and LY17H030012), the Anesthesiology Center in North of Zhejiang Province and the Jiaxing Key Laboratory of Neurology and Pain Medicine (2015-02-2).

\section{References}

1. Jemal A, Bray F, Center MM, Ferlay J, Ward E and Forman D: Global cancer statistics. CA Cancer J Clin 61: 69-90, 2011.

2. Liaw YF, Kao JH, Piratvisuth T, Chan HL, Chien RN, Liu CJ, Gane E, Locarnini S, Lim SG, Han KH, et al: Asian-Pacific consensus statement on the management of chronic hepatitis B: A 2012 update. Hepatol Int 6: 531-561, 2012.

3. Maluccio M and Covey A: Recent progress in understanding, diagnosing, and treating hepatocellular carcinoma. CA Cancer J Clin 62: 394-399, 2012.

4. Zhu GQ, Shi KQ, Yu HJ, He SY, Braddock M, Zhou MT, Chen YP and Zheng MH: Optimal adjuvant therapy for resected hepatocellular carcinoma: A systematic review with network meta-analysis. Oncotarget 6: 18151-18161, 2015.

5. de Lau WB, Snel B and Clevers HC: The R-spondin protein family. Genome Biol 13: 242, 2012.

6. Nam JS, Turcotte TJ and Yoon JK: Dynamic expression of R-spondin family genes in mouse development. Gene Expr Patterns 7: 306-312, 2007.

7. Parma P, Radi O, Vidal V, Chaboissier MC, Dellambra E, Valentini S, Guerra L, Schedl A and Camerino G: R-spondin1 is essential in sex determination, skin differentiation and malignancy. Nat Genet 38: 1304-1309, 2006.

8. Bell SM, Schreiner CM, Wert SE, Mucenski ML, Scott WJ and Whitsett JA: R-spondin 2 is required for normal laryngeal-tracheal, lung and limb morphogenesis. Development 135: 1049-1058, 2008.

9. Aoki M, Mieda M, Ikeda T, Hamada Y, Nakamura H and Okamoto H: R-spondin3 is required for mouse placental development. Dev Biol 301: 218-226, 2007.

10. Khan TN, Klar J, Nawaz S, Jameel M, Tariq M, Malik NA, Baig SM and Dahl N: Novel missense mutation in the RSPO4 gene in congenital hyponychia and evidence for a polymorphic initiation codon (p.M1I). BMC Med Genet 13: 120, 2012. 
11. Lowther W, Wiley K, Smith GH and Callahan R: A new common integration site, Int7, for the mouse mammary tumor virus in mouse mammary tumors identifies a gene whose product has furin-like and thrombospondin-like sequences. J Virol 79: 10093-10096, 2005 .

12. Theodorou V, Kimm MA, Boer M, Wessels L, Theelen W, Jonkers J and Hilkens J: MMTV insertional mutagenesis identifies genes, gene families and pathways involved in mammary cancer. Nat Genet 39: 759-769, 2007.

13. Kim KA, Kakitani M, Zhao J, Oshima T, Tang T, Binnerts M, Liu Y, Boyle B, Park E, Emtage P, et al: Mitogenic influence of human R-spondin1 on the intestinal epithelium. Science 309: 1256-1259, 2005.

14. Seshagiri S, Stawiski EW, Durinck S, Modrusan Z, Storm EE, Conboy CB, Chaudhuri S, Guan Y, Janakiraman V, Jaiswal BS, et al: Recurrent R-spondin fusions in colon cancer. Nature 488: 660-664, 2012

15. Shinmura K, Kahyo T, Kato H, Igarashi H, Matsuura S, Nakamura S, Kurachi K, Nakamura T, Ogawa H, Funai K, et al: RSPO fusion transcripts in colorectal cancer in Japanese population. Mol Biol Rep 41: 5375-5384, 2014.

16. Kim KA, Wagle M, Tran K, Zhan X, Dixon MA, Liu S, Gros D, Korver W, Yonkovich S, Tomasevic N, et al: R-Spondins family members regulate the Wnt pathway by a common mechanism. Mol Biol Cell 19: 2588-2596, 2008.

17. Huch M, Dorrell C, Boj SF, van Es JH, Li VS, van de Wetering M, Sato T, Hamer K, Sasaki N, Finegold MJ, et al: In vitro expansion of single $\mathrm{Lgr}^{+}$liver stem cells induced by Wnt-driven regeneration. Nature 494: 247-250, 2013.

18. Carmon KS, Gong X, Yi J, Thomas A and Liu Q: RSPO-LGR4 functions via IQGAP1 to potentiate Wnt signaling. Proc Natl Acad Sci USA 111: E1221-E1229, 2014.

19. Logan CY and Nusse R: The Wnt signaling pathway in development and disease. Annu Rev Cell Dev Biol 20: 781-810, 2004.

20. Clevers $\mathrm{H}$ : Wnt/beta-catenin signaling in development and disease. Cell 127: 469-480, 2006.

21. Monga SP: $\beta$-Catenin signaling and roles in liver homeostasis, injury and tumorigenesis. Gastroenterology 148: 1294-1310, 2015.
22. Stewart DJ: Wnt signaling pathway in non-small cell lung cancer J Natl Cancer Inst 106: djt356, 2014.

23. Gregorieff A, Liu Y, Inanlou MR, Khomchuk Y and Wrana JL Yap-dependent reprogramming of $\operatorname{Lgr5}(+)$ stem cells drives intestinal regeneration and cancer. Nature 526: 715-718, 2015.

24. Hu T and Li C: Convergence between Wnt- $\beta$-catenin and EGFR signaling in cancer. Mol Cancer 9: 236, 2010.

25. Ahn SM, Jang SJ, Shim JH, Kim D, Hong SM, Sung CO, Baek D, Haq F, Ansari AA, Lee SY, et al: Genomic portrait of resectable hepatocellular carcinomas: Implications of RB1 and FGF19 aberrations for patient stratification. Hepatology 60: 1972-1982, 2014.

26. Kononen J, Bubendorf L, Kallioniemi A, Bärlund M, Schraml P, Leighton S, Torhorst J, Mihatsch MJ, Sauter G and Kallioniemi OP: Tissue microarrays for high-throughput molecular profiling of tumor specimens. Nat Med 4: 844-847, 1998

27. Boseman FT, Carneiro F, Hruban RH and Theise ND (eds): Tumours of the liver and intrahepatic bile ducts. In: World Health Organization Classification of Tumours of the Digestive System. 4th edition. IARC, Lyon, pp195-262, 2010.

28. Kang N, Gores GJ and Shah VH: Hepatic stellate cells: Partners in crime for liver metastases? Hepatology 54: 707-713, 2011.

29. Coulouarn C and Clément B: Stellate cells and the development of liver cancer: Therapeutic potential of targeting the stroma. J Hepatol 60: 1306-1309, 2014.

30. Xinguang Y, Huixing Y, Xiaowei W, Xiaojun W and Linghua Y: R-spondin1 arguments hepatic fibrogenesis in vivo and in vitro. J Surg Res 193: 598-605, 2015

31. Yin X, Yi H, Wu W, Shu J, Wu X and Yu L: R-spondin2 activates hepatic stellate cells and promotes liver fibrosis. Dig Dis Sci 59: 2452-3961, 2014.

32. Chartier C, Raval J, Axelrod F, Bond C, Cain J, Dee-Hoskins C, Ma S, Fischer MM, Shah J, Wei J, et al: Therapeutic targeting of tumor-derived R-spondin attenuates $\beta$-catenin signaling and tumorigenesis in multiple cancer types. Cancer Res 76: 713-722, 2016. 